\title{
Clinical utility gene card for: MUTYH-associated polyposis (MAP), autosomal recessive colorectal adenomatous polyposis
}

\author{
Stefan $\operatorname{Aretz}^{\star, 1}$ and Frederik J Hes ${ }^{2}$ \\ European Journal of Human Genetics (2010) 18, doi:10.1038/ejhg.2010.77; published online 26 May 2010
}

\section{DISEASE CHARACTERISTICS}

1.1 Name of the disease (synonyms)

MUTYH-associated polyposis (MAP), autosomal recessive colorectal adenomatous polyposis.

\subsection{OMIM\# of the disease 608456.}

1.3 Name of the analysed genes or DNA/chromosome segments MUTYH (MYH, outdated name of the gene).

1.4 OMIM\# of the gene(s)

604933.

\subsection{Mutational spectrum}

Broad, all types of point mutations (missense, splice site, and truncating mutations). Gross genomic deletions or duplications have not been described yet. ${ }^{1-4}$

The two hotspot mutations Y179C (previously annotated as Y165C) in exon 7 and G396D (previously annotated as G382D) in exon 13 dominate in populations of European origin (together they account for around $80 \%$ of all reported mutant alleles). Thus, missense mutations are the predominant mutation type in Caucasian patients.

Mutations were described in almost all exons (except exons 1 and 2).

In parts ethnicity-specific mutational spectrum.

\subsection{Analytical methods}

Direct sequencing of all 16 exons (standard approach in routine diagnostics).

Screening the complete gene: DHPLC, SSCP, HD, CSGE, melting curve analysis.

Screening for both hotspot mutations (useful only in patients of European origin): restriction digest, ARMS, pyrosequencing.

Some procedures start with a screening for both hotspot mutations. If only one of them is detected, the whole gene is searched for a second mutation. By this method a considerable proportion (up to 20\%) of biallelic mutation carriers is missed. ${ }^{1}$

\subsection{Analytical validation}

The results of molecular genetic diagnostics can, as a rule, be definitely evaluated.

\subsection{Estimated Frequency of the Disease}

(Incidence at birth ('birth prevalence') or population prevalence) The frequency of MAP is around $15-20 \%$ in unselected APC mutation-negative patients with colorectal adenomatous polyposis of European origin.

The prevalence in population-based CRC patients of European origin is around $0.4 \%$. Assuming a CRC frequency of $5 \%$ in the general population, the overall prevalence is assumed to be around $1: 5000$. Of note, up to one third of biallelic MUTYH mutation carriers identified in population-based CRC studies developed CRC without a colorectal polyposis. ${ }^{5}$

From the frequency of MUTYH heterozygotes in the general population $(\sim 2 \%)$ the MAP prevalence (clinical plus subclinical biallelic carriers) can be derived as about 1:10000; however, the penetrance of biallelic MUTYH mutations is yet unknown. ${ }^{3}$

1.9 If applicable, prevalence in the ethnic group of investigated person

The known ethnicity-specific mutation patterns are not sufficient to derive ethnicity-specific prevalence rates. However, due to the presence of founder mutations among ethnic groups differences in the frequency of MAP are likely to exist. The two hotspot mutations Y179C and G396D observed in patients of European origin are almost absent in Asian populations.

\subsection{Diagnostic setting}

\begin{tabular}{lll}
\hline & Yes. & No. \\
A. (Differential) diagnostics & $\Downarrow$ & $\square$ \\
B. Predictive testing & $\Downarrow$ & $\square$ \\
C. Risk assessment in relatives & $\Downarrow$ & $\square$ \\
D. Prenatal & $\square$ & $\square$ \\
\hline
\end{tabular}

\footnotetext{
${ }^{1}$ Institute of Human Genetics, University of Bonn, Bonn, Germany; ${ }^{2}$ Department of Clinical Genetics, Leiden University Medical Center, Leiden, The Netherlands ${ }^{*}$ Correspondence: PD Dr S Aretz, Institute of Human Genetics, University of Bonn, Biomedical Center, Sigmund-Freud-Str. 25, Bonn, NRW 53127, Germany.

Tel: +49 228287 51009; Fax: +49 228287 51011; E-mail: Stefan.Aretz@uni-bonn.de
} 


\section{Comment:}

A prenatal diagnosis is almost never requested and, given a relatively late manifest and treatable disease, should be performed only exceptionally, in reasonable cases with clear indication and after extensive human genetic counselling.

\section{TEST CHARACTERISTICS}

\begin{tabular}{|c|c|c|c|c|}
\hline & \multicolumn{2}{|c|}{ Genotype or disease } & \multirow{2}{*}{$\begin{array}{l}\text { A: true positives } \\
\text { B: false positives }\end{array}$} & \multirow{2}{*}{$\begin{array}{l}\text { C: false negative } \\
\text { D: true negative }\end{array}$} \\
\hline & Present & Absent & & \\
\hline \multicolumn{5}{|l|}{ Test } \\
\hline \multirow[t]{2}{*}{ Positive } & A & B & Sensitivity: & $A /(A+C)$ \\
\hline & & & Specificity: & $D /(D+B)$ \\
\hline \multirow[t]{2}{*}{ Negative } & C & $\mathrm{D}$ & Positive predictive value: & $A /(A+B)$ \\
\hline & & & Negative predictive value: & $\mathrm{D} /(\mathrm{C}+\mathrm{D})$ \\
\hline
\end{tabular}

\subsection{Analytical sensitivity}

(proportion of positive tests if the genotype is present)

Almost 100\% (by sequencing of all 16 exons).

\subsection{Analytical specificity}

(proportion of negative tests if the genotype is not present) Almost 100\%.

\subsection{Clinical sensitivity}

(proportion of positive tests if the disease is present)

The clinical sensitivity can be dependent on variable factors such as age or family history. In such cases a general statement should be given, even if a quantification can only be made case by case.

About $15-20 \%$ (depending on severity of disease and family history: up to $60 \%$ with clear recessive inheritance).

\subsection{Clinical specificity}

(proportion of negative tests if the disease is not present)

The clinical specificity can be dependent on variable factors such as age or family history. In such cases a general statement should be given, even if a quantification can only be made case by case.

Almost $100 \%$ (except casually uncertain variants).

\subsection{Positive clinical predictive value}

\section{(lifetime risk to develop the disease if the test is positive)}

Penetrance in proven biallelic mutation carriers, according to present knowledge, is up to $100 \%$. Owing to clinical variability, mildly affected persons may not be diagnosed or they died from other reasons during presymptomatic (subclinical) stage of the disease.

\subsection{Negative clinical predictive value}

(Probability not to develop the disease if the test is negative, although the population risk for CRC and adenomas remains) Assume an increased risk based on family history for a non-affected person. Allelic and locus heterogeneity may need to be considered.

Index case in that family had been tested:

Almost $100 \%$ for sibs of index patient; slightly less for obligate heterozygous children because the mutational status of the other parent probably cannot be assessed with $100 \%$ certainty. Detection of an uncertain variant in the child of an affected person will not allow in some cases a secure decision whether the test is 'negative'. De novo mutations have not been described.
Index case in that family had not been tested:

Probably about $5-10 \%$ if index case has not been tested for APC mutation either (locus heterogeneity), but also depending on family history (dominant $v s$ recessive pedigree pattern).

This is not a meaningful approach and should therefore be avoided. Particularly in attenuated courses of adenomatous polyposis more and still unknown genes may be involved.

\section{CLINICAL UTILITY}

3.1 (Differential) diagnosis: the tested person is clinically affected (To be answered if in 1.10 'A' was marked)

MUTYH mutation analysis should be considered in patients with multiple $(>10)$ synchronous colorectal adenomas of rather late onset in the absence of a clear autosomal dominant inheritance pattern. As hyperplastic polyps and sessile serrated adenomas are a common finding in MAP and the occurrence of CRC in the absence of colorectal polyps was described, the clinical presentation might be misdiagnosed as hyperplastic polyposis or sporadic CRC in some patients. ${ }^{5,6} \mathrm{~A}$ phenotypic overlap with HNPCC (Lynch syndrome) was reported. ${ }^{7}$

In population-based and early onset CRC cohorts and in patients with solely a few adenomas at any age, without an overt family history (and no carcinoma), the frequency of biallelic MUTYH mutations is low $(0.3-1.7 \%)$. For these cases currently no general recommendations regarding MUTYH mutation analysis exist.

\subsubsection{Can a diagnosis be made other than through a genetic test?}

\begin{tabular}{ll}
\hline No $\quad \square$ (continue with 3.1.4) \\
Yes $\quad \square$ \\
Clinically \\
Imaging \\
Endoscopy \\
Biochemistry \\
Electrophysiology \\
Other (please describe) In some rare cases the condition can be assumed \\
by family history (typical autosomal recessive inheritance pattern). \\
However, the diagnosis must be confirmed by identification of a biallelic \\
MUTYH mutation. In the majority of patients differentiating MAP from \\
APC-associated familial adenomatous polyposis (FAP) can be achieved by \\
molecular genetic analysis only.
\end{tabular}

3.1.2 Describe the burden of alternative diagnostic methods to the patient Family history: no strain.

3.1.3 How is the cost effectiveness of alternative diagnostic methods to be judged?

Family history: very cost-effective.

3.1.4 Will disease management be influenced by the result of a genetic test?

No. $\otimes$

Yes.

3.2 Predictive setting: the tested person is clinically unaffected but carries an increased risk based on family history

(To be answered if in 1.10 'B' was marked)

\subsubsection{Will the result of a genetic test influence lifestyle and prevention?} Yes

If the test result is positive (please describe):

Yes. Heightened motivation of siblings to participate in specific preventive checkups, eventually colectomy when adenomas etc. are detected. 
In some cases family planning, choice of profession.

To subject children of affected persons to early diagnosis programs appears useful only if, by predictive testing, they were shown to be biallelic carriers.

If the test result is negative (please describe):

Yes.

Release from preventive program, psychological relief.

3.2.2 Which option in view of lifestyle and prevention does a person at risk have if no genetic test has been done (please describe)?

Same for siblings of index patients as for proven biallelic carriers: close-meshed early diagnosis and surveillance programs (particularly frequent complete colonoscopies).

Children of affected persons, because of their low disease risk $(\sim 1 \%)$, should undergo an intensive early diagnosis program only if they are proven biallelic carriers. Whether with the comparatively low a priori disease risk of children predictive diagnostics should be offered at all remains, for the present, a matter of discretion.

So far, one study suggested that it may be appropriate to initiate surveillance later for p.G396D homozygotes and p.G396D/p.Y179C compound heterozygotes as compared with p.Y179C homozygotes. ${ }^{8}$

3.3 Genetic risk assessment in family members of a diseased person (To be answered if in 1.10 ' $\mathrm{C}$ ' was marked)

\subsubsection{Does the result of a genetic test resolve the genetic situation in} that family?

Yes.

\subsubsection{Can a genetic test in the index patient save genetic or other tests} in family members?

Yes, by securing the primary cause of the disease, extended diagnostic investigations in other symptomatic relatives can be avoided.

The low recurrence risk in children and exclusion of carriership by predictive diagnostics in siblings of affected persons allow to avoid superfluous preventive checkups and offer psychological relief.

3.3.3 Does a positive genetic test result in the index patient enable a predictive test in a family member?

Yes.

\subsection{Prenatal diagnosis}

(To be answered if in 1.10 ' $\mathrm{D}$ ' was marked)
3.4.1 Does a positive genetic test result in the index patient enable a prenatal diagnostic?

Yes (but see the comment in 2.10).

\section{IF APPLICABLE, FURTHER CONSEQUENCES OF TESTING}

Please assume that the result of a genetic test has no immediate medical consequences. Is there any evidence that a genetic test is nevertheless useful for the patient or his/her relatives? (Please describe)

For many patients prove of diagnosis is a value in itself irrespective of a medical benefit - because the disease and its cause can often clearly be named.

When a genetic cause is verified, an assumption of 'own fault' as cause of disease (exogenous poisons, 'wrong conduct') often can be lapsed with relief.

The main benefits of genetic diagnostics in MAP are the differentiation from FAP, a precise recurrence risk for close relatives, and relief of non-carriers during predictive diagnostics.

\section{CONFLICT OF INTEREST}

The authors declare no conflict of interest.

\section{ACKNOWLEDGEMENTS}

This work was supported by EuroGentest, an EU-FP6-supported NoE, contract number 512148 (EuroGentest Unit 3: 'Clinical genetics, community genetics and public health', Workpackage 3.2)

1 Aretz S, Uhlhaas S, Goergens H et al: MUTYH-associated polyposis (MAP): 70 of 71 patients with biallelic mutations present with an attenuated or atypical phenotype. Int J Cancer 2006; 119: 807-814.

2 Poulsen ML, Bisgaard ML: MUTYH associated polyposis (MAP). Curr Genomics 2008; 9: 420-435.

3 Sampson JR, Jones N: MUTYH-associated polyposis. Best Pract Res Clin Gastroenterol 2009; 23: 209-218.

4 Molatore S, Russo MT, D'Agostino VG et al: MUTYH mutations associated with familial adenomatous polyposis: functional characterization by a mammalian cell-based assay. Hum Mutat 2010; 31: 159-166.

5 Cleary SP, Cotterchio M, Jenkins MA et al: Germline MutY human homologue mutations and colorectal cancer: a multisite case-control study. Gastroenterology 2009; 136: $1251-1260$.

6 Boparai KS, Dekker E, Van Eeden S et al: Hyperplastic polyps and sessile serrated adenomas as a phenotypic expression of MYH-associated polyposis. Gastroenterology 2008; 135: 2014-2018.

7 Vogt S, Jones N, Christian D et al: Expanded extracolonic tumor spectrum in MUTYH associated polyposis. Gastroenterology 2009; 137: 1976-1985.

8 Nielsen M, Joerink-van de Beld MC, Jones N et al: Analysis of MUTYH genotypes and colorectal phenotypes in patients with MUTYH-associated polyposis. Gastroenterology 2009; 136: 471-476. 